\title{
Infographic: Physical Activity for Pregnant Women
}

First author and Correspondence to:

Dr Ralph Smith, Nuffield Department of Population Health, University of Oxford, UK Ralph.Smith@doctors.org.uk

Co authors:

Hamish Reid ${ }^{1}$

Anne Matthews ${ }^{1}$

Catherine Calderwood ${ }^{2}$

Marian Knight ${ }^{3,4}$

Charlie Foster ${ }^{4,5}$

1. Nuffield Department of Population Health, University of Oxford, UK

2. Chief Medical Officer for Scotland, Edinburgh, UK

3. National Perinatal Epidemiology Unit NPEU, Oxford, Oxfordshire, UK

4. On behalf of $\mathrm{CMO}$ Physical Activity Expert Committee for Physical Activity and Pregnancy

5. University of Bristol Centre for Exercise, Nutrition and Health Sciences, School for Policy Studies, Faculty of Social Sciences and Law, Bristol, UK 
Our recent BJSM editorial petitioned for 'a time for action' regarding physical activity (PA) and pregnancy guidance in the UK. [1] We highlighted that despite pregnancy providing a unique opportunity to promote PA behaviour change, [2] healthcare professionals (HPs) lack the confidence, knowledge and resources to deliver appropriate PA advice. They feel under resourced, uncertain regarding safety concerns and inadequately trained. [3] Consequently, despite being receptive to PA advice, pregnant women receive inconsistent, vague and conflicting guidance. [4] These issues may contribute to reduced levels of physical activity during pregnancy, leaving few women meeting the recommended activity levels. [5]

The 2011 UK Chief Medical officers (CMOs') PA recommendations led to the development of a series of simple evidence based infographics designed to empower HPs to promote PA behaviour change across the life course. $[6,7]$ Remarkably, pregnant women were the only group of the population omitted from the Chief Medical Officers' 2011 PA recommendations. To address this, the four CMOs tasked an PA Expert Committee to firstly review the available evidence and, if sufficient, subsequently provide evidence-based recommendations in the form of an infographic.

We reviewed a variety of evidence sources. Firstly, we conducted a systematic review of reviews of the epidemiological evidence related to PA and pregnancy outcomes. Subsequently, we conducted a structured review of key qualitative studies, current grey literature and relevant existing infographics. Finally, we sought expert professional opinions using the FUSE Network (Centre for Translational Research in Public Health). The committee developed an infographic which was subsequently field-tested with over $250 \mathrm{HPs}$. The final draft was subsequently submitted to the CMOs for final editing and approval.

The infographic is the final product of this process and is the latest addition to the series. It aims to provide clarity and a consistency to equip HPs to deliver evidence-based recommendations to enhance antenatal care, allowing women the confidence to experience the benefits of being active throughout pregnancy and beyond. It provides objective details regarding intensity, time and frequency. It aims to convey the positive message of encouraging women to incorporate PA into their daily routines with specific examples of 
suitable activity underpinned by key primary safety messages. These specific benefits include reducing the risk of gestational diabetes, excessive gestational weight gain and improving cardiovascular fitness. These benefits are in addition to those gained by following the adult recommendations. [8] It is important to note, we found no systematic reviews that identified any adverse maternal or infant outcomes resulting from PA during pregnancy [8] adding reassurance to any safety concerns.

Similar to the other CMO Physical Activity infographics, this resource has been designed to help health professionals to facilitate effective PA counselling and aid consultations. Unique to this infographic, there is an additional link which provides HCPs with further details and the supporting evidence presented in a guidance document.

We urge all readers to share this infographic across their professional networks empowering HPs to advise patients on PA throughout pregnancy with confidence and clarity ensuring best clinical practice. Encouraging physical activity during pregnancy will provide evidence based benefits. This infographic can support these interventions, which in turn may provide an active springboard for families to sustain healthy behaviours beyond childbirth.

Word Count: 531 


\section{Competing Interests}

All authors were part of the group developing this infographic.

\section{Contributorship Statement:}

We can confirm that all Author have had substantial contributions to the conception or design of this work.

\section{Funding}

There is no funding to report for this submission

Data Sharing Statement:

The infographic is available via the weblink below with an additional guidance link. All available data can be obtained by contacting the corresponding author

https://www.gov.uk/government/publications/start-active-stay-active-infographics-onphysical-activity

Ethical approval information:

This is an editorial. No human subjects were involved. Therefore Ethics committee/institutional review board approval was not obtained. 


\section{References:}

1. Reid H, Smith R, Calderwood C, Foster C. Physical activity and pregnancy: time for guidance in the UK. Br J Sports Med. 2017.

2. ACOG Committee Opinion No. 650: Physical Activity and Exercise During Pregnancy and the Postpartum Period. Obstet Gynecol. 2015;126(6):e135-42.

3. Brown MJ SM, Liddle D, Hill AJ,, Stockdale J ME. Motivating pregnant women to eat healthily and engage in physical activity for weight management: an exploration of routine midwife instruction. Evidence Based Midwifery. 2013;11(4):120-7.

4. Weir Z, Bush J, Robson SC, McParlin C, Rankin J, Bell R. Physical activity in pregnancy: a qualitative study of the beliefs of overweight and obese pregnant women. BMC Pregnancy Childbirth. 2010;10:18.

5. Borodulin KM, Evenson KR, Wen F, Herring AH, Benson AM. Physical activity patterns during pregnancy. Med Sci Sports Exerc. 2008;40(11):1901-8.

6. Reid H, Davies S, Calderwood C, Atherton F, McBride M, Foster C. Infographic. Physical activity for early years. Br J Sports Med. 2016.

7. Reid H, Foster C. Infographic. Physical activity benefits for adults and older adults. Br J Sports Med. 2016.

8. University of Oxford Physical Activity and Pregnancy Study Group. Physical Activity and Pregnancy Infographic Study Interim Report. 2016. Unpublished work. University of Oxford, Oxford. 